\title{
CT findings of 795 COVID-19 positive cases: a multicenter study in Egypt
}

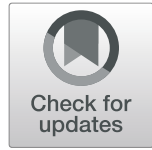

\author{
Youssriah Yahia Sabri ${ }^{1}$, Mohamed Mohsen Tolba Fawzi ${ }^{2}$, Eman Zaki Nossair ${ }^{2 *}$ (D, Safaa Mohamed El-Mandooh², \\ Amira Aly Hegazy ${ }^{1}$ and Sally Fouad Tadros ${ }^{1}$
}

\begin{abstract}
Background: Corona Virus Disease 2019 (COVID-19) outbreak was officially announced as a global pandemic by the WHO on March $11^{\text {th }}$ 2020. Thorough understanding of CT imaging features of COVID-19 is essential for effective patient management; rationalizing the need for relevant research. The aim of this study was to analyze the chest $C T$ findings of patients with real-time polymerase chain reaction (RT-PCR) proved COVID-19 admitted to four Egyptian hospitals. The recently published RSNA expert consensus statement on reporting COVID-19 chest CT findings was taken into consideration.

Results: Normal CT "negative for COVID-19" was reported in 26.1\% of our RT-PCR proved COVID-19 cases. In descending order of prevalence, imaging findings of the positive CT studies (73.9\%) included GGO (69\%), consolidation (49.7\%), crazy paving (15.4\%), and peri-lobular fibrosis (40.6\%). These showed a dominantly bilateral (68.2\%), peripheral (72.4\%), and patchy (64.7\%) distribution. Remarkably, thymic hyperplasia was identified in $14.3 \%$ of studies. According to the RSNA consensus, CT findings were classified as typical in 68.9\%, indeterminate in 3.6\%, and atypical in $1.4 \%$ of the evaluated CT studies.
\end{abstract}

Conclusion: Although COVID-19 cannot be entirely excluded by chest $C T$, it can be distinguished in more than two-thirds of cases; making CT a widely available, non-invasive, and rapid diagnostic tool.

Keywords: COVID-19;Chest CT, Egypt

\section{Background}

Coronavirus Disease 2019 (COVID-19) outbreak caused by "severe acute respiratory syndrome coronavirus 2" (SARS-CoV-2) was officially announced as a global pandemic by the World Health Organization (WHO) on March 11, $2020[1,2]$. A total of 7,553,182 cases and 423,349 deaths had been reported worldwide by June 13, 2020, from which 41,303 cases and 1,422 deaths recorded in Egypt [3].

COVID-19 infection may be asymptomatic, may present with mild non-specific symptoms such as fever, cough, or fatigue, and may progress to severe symptoms

\footnotetext{
* Correspondence: eman.zaki5@gmail.com

${ }^{2}$ National Hepatology \& Tropical Medicine Research Institute (NHTMRI), 10 (A) Kasr El-Aini St, Cairo 11796, Egypt

Full list of author information is available at the end of the article
}

including respiratory failure or even death in patients of old age and/or other co-morbidities [4].

The gold standard for diagnosing COVID-19 up till now is a positive nucleic acid testing (NAT) using reverse-transcriptase polymerase-chain-reaction (RTPCR) [1]. Several papers reported the typical COVID-19 chest findings as multifocal bilateral ground glass opacities (GGOs) with or without patchy consolidations in a peripheral subpleural distribution and posterior lower lobe predilection [4]. However, further studies found other findings including crazy paving pattern, airway changes, reversed halo sign, etc. [5]. CT is more efficient in detection of GGO than radiography [4]. Thus various studies advocated the use of chest CT in the management of COVID-19 patients [2, 6, 7].

Four categories were proposed by the Radiological Society of North America (RSNA) Expert Consensus

\section{Springer Open}

(c) The Author(s). 2020 Open Access This article is licensed under a Creative Commons Attribution 4.0 International License, which permits use, sharing, adaptation, distribution and reproduction in any medium or format, as long as you give appropriate credit to the original author(s) and the source, provide a link to the Creative Commons licence, and indicate if changes were made. The images or other third party material in this article are included in the article's Creative Commons licence, unless indicated otherwise in a credit line to the material. If material is not included in the article's Creative Commons licence and your intended use is not permitted by statutory regulation or exceeds the permitted use, you will need to obtain permission directly from the copyright holder. To view a copy of this licence, visit http://creativecommons.org/licenses/by/4.0/. 
Statement on reporting chest CT Findings related to COVID-19 depending on the type of lesion encountered: (1) typical features which are observed frequently and more distinctly in COVID-19 pneumonia, (2) indeterminate features which are observed in COVID-19 pneumonia but are not characteristic, (3) atypical features which are infrequently observed in COVID-19 pneumonia and are more definitive of other infections, and (4) negative for pneumonia with no lung findings linked to infection, precisely, GGO and consolidation [2]. However, it should be recognized that chest CT may be negative early in COVID-19 [2].

The aim of this study was to analyze the chest CT findings of RT-PCR proved COVID-19 patients adopting the classification recommended by RSNA Expert Consensus Statement in order to test its diagnostic competence.

At that time, the health system in Egypt was still capable of isolating all PCR-confirmed cases of COVID-19 regardless of disease severity including asymptomatic contacts; to limit infection spread in the context of that phase of the pandemic [8].

\section{Methods}

\section{Study population and design}

Before conducting this prospective study, local institutional review board (IRB) approval was granted. Written informed consent was obtained from all study participants or their authorized representatives.
This study initially involved 795 consecutive participants admitted to four Egyptian hospitals in the period from March 2, 2020 to June 13, 2020. All subjects had RT-PCR confirmed COVID-19 and underwent chest CT upon admission. Only those whose CT was degraded by motion artifacts were excluded $(n=23)$. Thereby, a total of 772 participants were enrolled in the study [431 males (age range, 4 months-95 years; mean, 45.66 years) and 341 females (age range, 3-95 years; mean, 44.11 years)] (Fig. 1).

All relevant clinical, laboratory, and epidemiologic data were provided by the admitting physicians.

\section{Imaging technique/acquisition}

Participants were scanned with the following scanners: Aquilion Lightning "16-row 32 slice" (Toshiba Medical Systems) and Aquilion Prime $^{\text {tw }}$ "80-row 160 slice" (Toshiba Medical Systems).

Acquisition parameters were set at $120 \mathrm{kVp} ; 100$ 200 mAs; pitch, 0.75-1.5; and collimation, 0.625-5 $\mathrm{mm}$. All imaging data were reconstructed using a medium sharp reconstruction algorithm with a slice thickness of $0.625-5 \mathrm{~mm}$.

With the patient in supine position, CT images were acquired at full inspiration from the level of the thoracic inlet to the diaphragm. No IV contrast was administered.

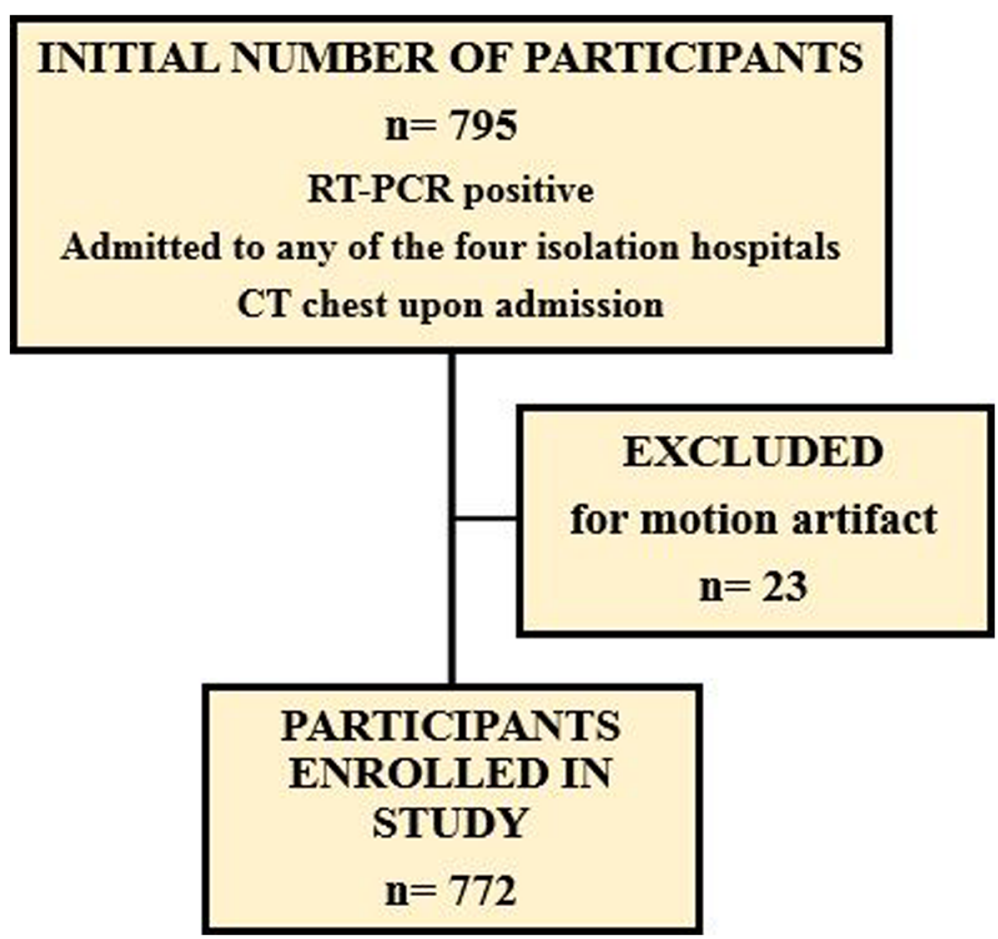

Fig. 1 Study flow chart 


\section{Imaging analysis}

The performed studies were transferred to PACS system (PaxeraUltima version 6.0.0.1 and MILLENSYS version 6.5.0.2579) for reviewing. Five radiologists with 9 to 32 years of experience (Y.Y.S., M.F.T, E.Z.N, S.M.E. and S.F.T.) interpreted the CT studies. Each study was reviewed by two of the five radiologists independently. In case of discrepancy, studies were re-reviewed by the thoracic radiologist with 32 years of experience (Y.Y.S.) then findings were discussed to reach a general agreement. Scans were viewed in both lung (WW/WL: 1500/ $-600 \mathrm{HU}$ ) and mediastinal (WW/WL 300/50 HU) windows.

Readers reported the presence of the following lung parenchymal findings: GGO, consolidation, crazy paving, peri-lobular fibrosis, reversed halo sign, vacuolar sign, pulmonary nodules, lobar pneumonia, and lung cavitation as well as associated traction bronchiectasis and vascular thickening. The involved lung lobes and lesions' pattern of distribution whether peripheral, peripheral and central, patchy, or diffuse were recorded. Subsequently, CT findings were allocated to one of the RSNA consensus categories. Other associated pulmonary, mediastinal, pleural, and upper abdominal CT findings were registered as well.

Seventy-one participants underwent follow-up CT studies to evaluate the progression or regression of the aforementioned findings in addition to newly developed observations-their scans were assessed accordingly.

\section{Statistical analysis}

The collected data were carefully revised, coded, tabulated, and introduced to a personal computer using "Microsoft Office Excel Software" program (2016) for windows by A.A.H. The pre-coded data were then transferred to the Statistical Package of Social Science Software program, version 23 to be statistically analyzed.

For qualitative variables, they were described as frequency and percentage. Comparison for qualitative variables was done by using chi-square test and Fisher's exact test, where $p$ value of significant correlation if $p<$ 0.05 .

Quantitative variables were presented using mean \pm standard deviation (SD). Comparison between groups was performed using independent $t$ test and analysis of variance (ANOVA) test followed by Bonferroni comparisons test. $P$ values less than 0.05 were considered statistically significant.

\section{Results}

This study enrolled 772 consecutive study participants [431 males and 341 females, age range 4 months-95 years, mean age $44.9 \pm 16.2$ years, inter-quartile range $32-58$ (26) years]. All participants presented to one of the four hospitals with positive RT-PCR tests. The patients' demographics and clinical data are listed in Table 1.

A total of 862 CT chest studies were performed including 772 initial and 90 follow up CT studies. On initial CT study, 569/772 (73.7\%) (age range 3 months-95 years; mean 49.84 years) had positive CT imaging manifestations of COVID-19, while 203/772 (26.3\%) (age range 3-95 years; mean 31.33 years) did not show any lung abnormalities despite a positive RT-PCR test. There was a statistically significant difference regarding age between those with positive and negative CT studies; where the ones with positive $\mathrm{CT}$ were older than the ones with negative CT studies ( $p$ value $<0.001$ ).

Male participants were more than females in both the positive $(304 / 569,53.4 \%)$ and negative $(127 / 203,62.6 \%)$ groups; however, it was not a statistically significant difference $(p$ value $=0.058)$ (Fig. 2).

Excluding 225/862 (26.1\%) normal chest CT studies, the imaging findings of the $637 / 862(73.9 \%)$ positive CT studies included ground glass opacities in 595/862 (69\%), consolidation in $428 / 862$ (49.7\%), crazy paving in $133 / 862$ (15.4\%), peri-lobular fibrosis in 350/862 (40.6\%), reversed halo sign in $231 / 862(26.8 \%)$, ground glass or sub-solid peripheral pulmonary nodules in 129/862 (15\%), lobar pneumonia in $3 / 862(0.3 \%)$ studies, and lung cavity in $1 / 862(0.1 \%)$ study only (Fig. 3$)$. Traction bronchiectasis was also observed in 302/862 (35\%), vascular thickening in 365/862 (42.3\%), and vacuolar sign in $69 / 862(8 \%)$ studies. Associated finding of pleural thickening was detected in $105 / 862(12.2 \%)$ studies; pleural effusion in 27/862 (3.1\%) studies; $22 / 862$ (2.6\%) bilateral, $4 / 862(0.5 \%)$ on the left side, and $1 / 862(0.1 \%)$ on the right side; pericardial effusion in $39 / 862$ (4.5\%) studies; lymphadenopathy in $47 / 862(5.5 \%)$ studies; $10 / 862$ (1.2\%) non-calcified and 37/862 (4.3\%) calcified; and

Table 1 Patients' demographics and clinical data

No. of patients (\%)

\begin{tabular}{ll}
\hline Patients' demographics: & \\
Number of CT studies & 862 \\
Number of patients & 772 \\
Mean age (years) & $44.98 \pm 16.21$ \\
Age range & 4 months-95 years \\
Interquartile range (years) & $32-58(26)$ \\
Males & $431(55.8 \%)$ \\
Females & $341(44.2 \%)$ \\
Clinical symptoms & \\
Fever & $581(75.3 \%)$ \\
Cough & $466(60.4 \%)$ \\
Dyspnea & $274(35.5 \%)$ \\
Asymptomatic contacts & $97(12.6 \%)$ \\
\hline
\end{tabular}




\section{MAIN LUNG CT FINDINGS IN COVID-19 PNEUMONIA}

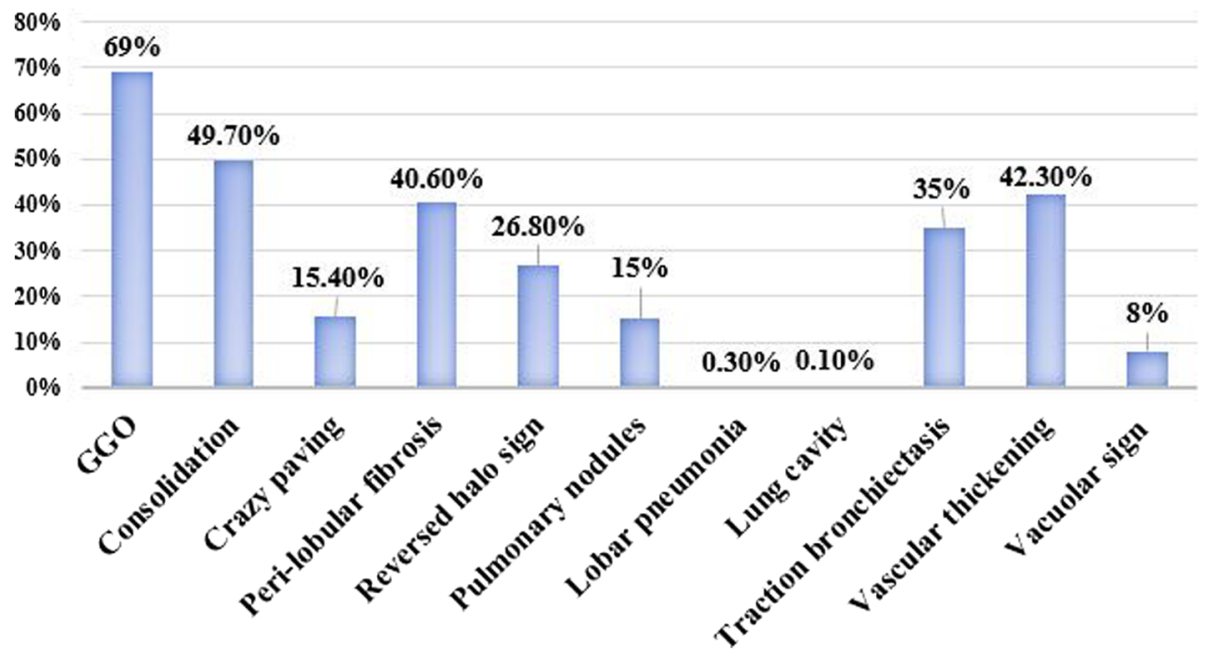

Fig. 2 Bar chart showing the prevalence of the main lung parenchymal CT findings in COVID-19 pneumonia

lung cysts in 7/862 (0.8\%) studies. Thymic hyperplasia [defined as retrosternal well-defined triangular-shaped soft tissue density with straight borders showing less macroscopic fat than expected for age [9] was identified in 123/862 (14.3\%) of studies (42 males and 81 females, age range 17-50 years; mean 26.1 years); out of which 102/862 (11.8\%) (37 males and 65 females, age range 21-50 years; mean 25.98 years) showed no CT lung findings of COVID-19 pneumonia with a statistically significant predilection for young females (Fig. 4). The prevalence of common and associated CT findings is reported in Tables 2 and 3 and Fig. 2.

Both lungs were involved in 588/862 (68.2\%), the right lung in $622 / 862(72.2 \%)$, and the left lung in $603 / 862$ $(70 \%)$ studies; the right upper lobe was implicated in $532 / 862(61.7 \%)$, middle lobe in 504/862 (58.5\%), right lower lobe in 609/862 (70.6\%), left upper lobe in 528/ 862 (61.3\%), lingula in 489/862 (56.7\%), and left lower

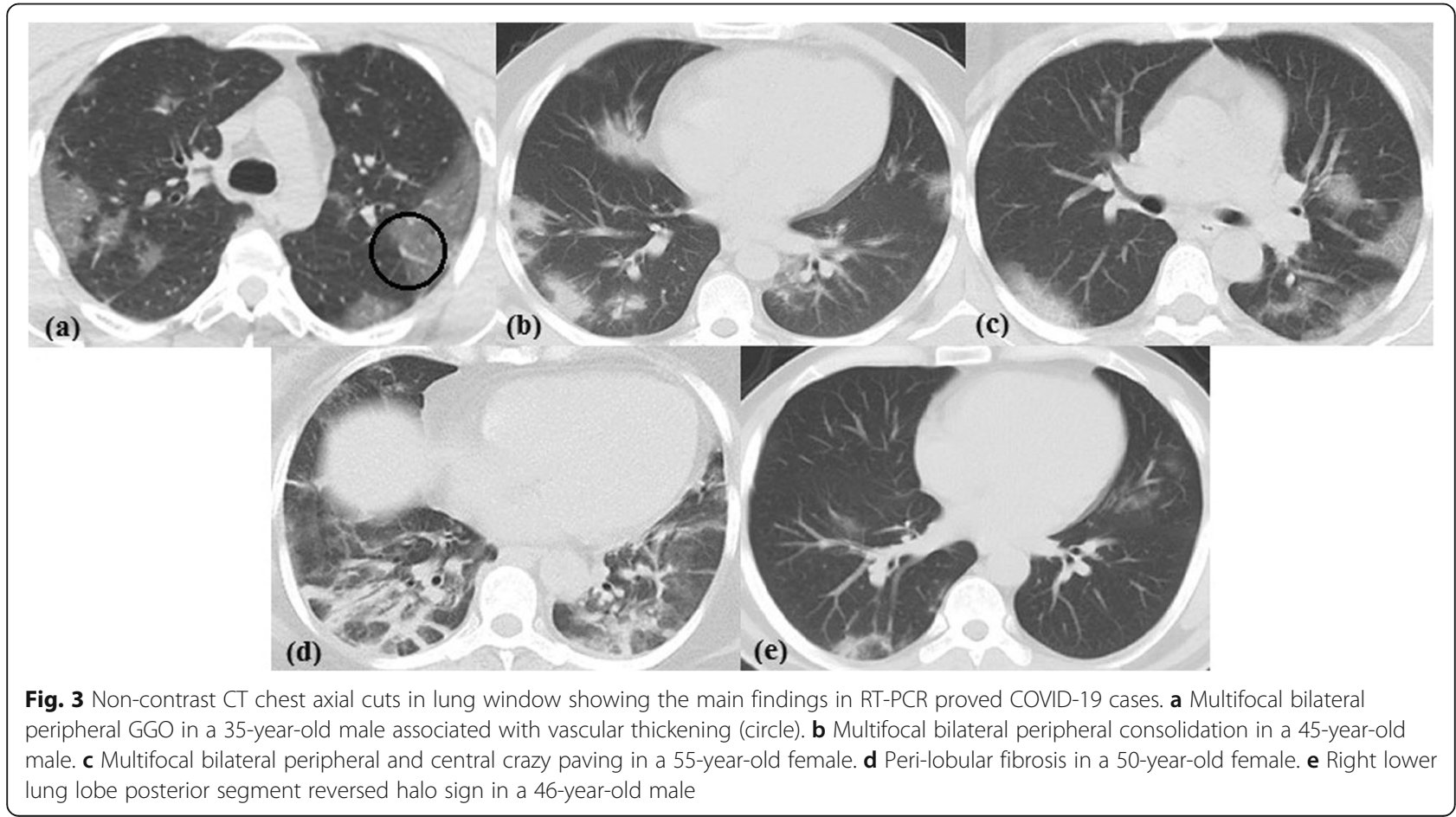




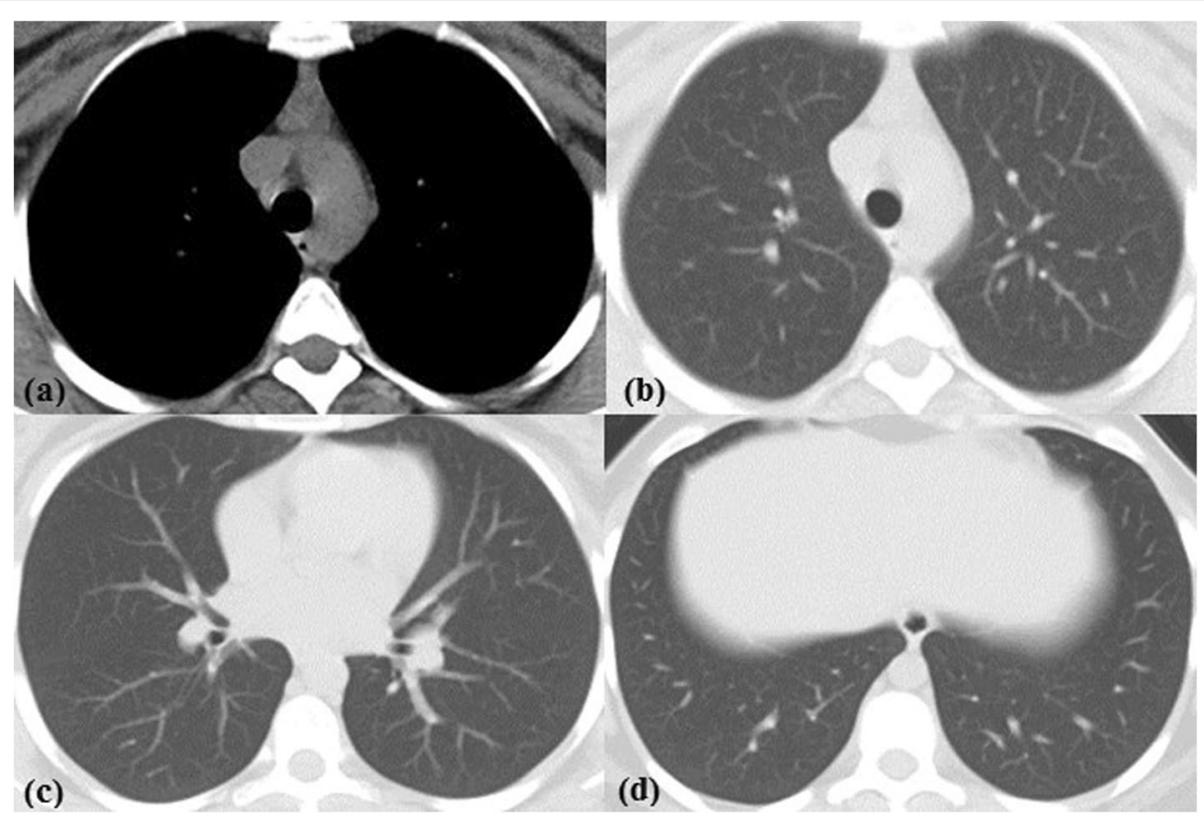

Fig. 4 Thymic hyperplasia in a 23-year-old female with RT-PCR proved COVID-19. a Non-contrast CT chest axial cut mediastinal window shows retrosternal well-defined triangular-shaped soft tissue density with straight borders with less macroscopic fat than expected for age. $\mathbf{b}$ - $\mathbf{d}$ Axial lung window showing no lung abnormality

lobe in 578/862 (67.1\%) studies. Unilateral lung affection was encountered in $49 / 862(5.7 \%)$ of studies while unilobar affection in $68 / 862(7.9 \%)$. The lesions showed peripheral distribution in 624/862 (72.4\%), both peripheral and central in $222 / 862(25.8 \%)$, patchy in $558 / 862$ (64.7\%), and diffuse in $16 / 862(1.9 \%)$ studies. The involved lung lobes and the lesions' distribution are recoded in Table 4.

According to the four categories endorsed by the RSNA Expert Consensus Statement, the CT findings in our study were reported as typical in 594/862 (68.9\%), indeterminate in $31 / 862$ (3.6\%), and atypical in $12 / 862$ (1.4\%) studies, while chest CT was normal; thus,

Table 2 Main CT imaging findings in COVID-19 pneumonia

\begin{tabular}{ll}
\hline Imaging finding & No. of patients (\%) \\
\hline Ground glass opacities & $595(69 \%)$ \\
Consolidation & $428(49.7 \%)$ \\
Crazy paving & $133(15.4 \%)$ \\
Peri-lobular fibrosis & $350(40.6 \%)$ \\
Reversed halo sign & $231(26.8 \%)$ \\
Peripheral subsolid pulmonary nodules & $129(15 \%)$ \\
Lobar pneumonia & $3(0.3 \%)$ \\
Lung cavity & $1(0.1 \%)$ \\
Traction bronchiectasis & $302(35 \%)$ \\
Vascular thickening & $365(42.3 \%)$ \\
Vacuolar sign & $69(8 \%)$ \\
\hline
\end{tabular}

reported as negative for COVID-19 pneumonia in 225/ 862 (26.1\%) studies.

Seventy-one participants underwent 90 follow-up CT studies according to clinical context (Fig. 5). Fifty-six performed 1 follow up, twelve performed 2 follow ups, two performed 3 follow ups, and one performed 4 follow ups. The time interval between the scans ranged from 2 to 49 days, with an average of 13.2 days.

Twenty-one participants showing normal initial CT underwent follow-up CT studies. One of them established imaging features of COVID-19 6 days after the initial CT, while the other 20/72 (27.8\%) (age range 2153 years; mean 32.65 years) had their follow up studies guided by a persistent positive RT-PCR at time interval ranging between 4 and 49 days from the initial to the follow up CT; yet, their chest CT studies remained normal on follow up.

The follow-up CT studies showed progression in 17/90 (18.9\%), regression in 44/90 (48.9\%), and stationary course in $29 / 90(32.2 \%)$ studies.

\section{Discussion}

COVID-19 pandemic has affected millions of people worldwide. Thorough comprehension of CT imaging features of COVID-19 is mandatory for effective patient management. Hence, we meticulously assessed the CT studies of 772 participants with RT-PCR proved COVID-19.

About one-quarter of them, with statistically significant younger age, showed normal initial CT study. This is consistent with the previous studies done by Ojha et al., Yang 
Table 3 Associated CT imaging findings in COVID-19 pneumonia

\begin{tabular}{|c|c|}
\hline Imaging finding & No. of patients (\%) \\
\hline \multicolumn{2}{|l|}{ Pulmonary } \\
\hline Old granulomatous infection (apical scarring/calcified nodules) & $73(8.47 \%)$ \\
\hline Cysts & $7(0.8 \%)$ \\
\hline Airway disease & $92(10.67 \%)$ \\
\hline \multicolumn{2}{|l|}{ Mediastinal } \\
\hline Lymphadenopathy: calcified/noncalcified & $47(5.5 \%) / 37(4.3 \%) / 10(1.2 \%)$ \\
\hline Pericardial effusion & $39(4.52 \%)$ \\
\hline Cardiomegaly & $123(14.27 \%)$ \\
\hline Pulmonary hypertension & $42(4.87 \%)$ \\
\hline Anterior mediastinal mass & $1(0.12 \%)$ \\
\hline Mediastinal bronchogenic cyst & $1(0.12 \%)$ \\
\hline Tracheal diverticulum & $5(0.58 \%)$ \\
\hline Thymic hyperplasia & $123(14.3 \%)$ \\
\hline Atheromatous vascular plaques & $111(12.88 \%)$ \\
\hline Ectatic ascending aorta & $17(1.97 \%)$ \\
\hline Lipomatosis & $1(0.12 \%)$ \\
\hline Median sternotomy sutures & $3(0.35 \%)$ \\
\hline Enlarged heterogeneous thyroid gland & $48(5.57 \%)$ \\
\hline Patulous esophagus & $3(0.35 \%)$ \\
\hline \multicolumn{2}{|l|}{ Pleural } \\
\hline Effusion & $27(3.1 \%)$ \\
\hline Thickening/reaction & $105(12.2 \%)$ \\
\hline Calcified plaques (Asbestos exposure) & $2(0.23 \%)$ \\
\hline \multicolumn{2}{|l|}{ Chest wall } \\
\hline Breast mass & $4(0.46 \%)$ \\
\hline \multicolumn{2}{|l|}{ Abdominal } \\
\hline Liver cirrhosis & $17(1.97 \%)$ \\
\hline Fatty liver & $69(8 \%)$ \\
\hline Hepatomegaly/Splenomegaly & $14(1.62 \%) / 13(1.51 \%)$ \\
\hline Hepatic/Splenic calcified focus & $9(1 \%)$ \\
\hline Hepatic/splenic focal lesion & $9(1 \%)$ \\
\hline Adrenal mass & $10(1.16 \%)$ \\
\hline Renal stone and backpressure changes & $21(2.4 \%)$ \\
\hline Renal cortical cyst & $14(1.62 \%)$ \\
\hline Renal atrophic changes & $3(0.35 \%)$ \\
\hline Ascites & $1(0.12 \%)$ \\
\hline Calcular gall bladder & $26(3.02 \%)$ \\
\hline
\end{tabular}

et al., Asefi and Safaie Xu et al., and Zhang et al. [6, 1013]. They stated that up to half of patients with positive RT-PCR may show a normal CT especially in the early phase or in asymptomatic infections. Therefore, CT cannot completely exclude COVID-19 infection.

In conformity with nearly all published studies [6, 7, $11,13-25]$, the most prevailing CT imaging finding of
COVID-19 in our population was GGO with or without consolidation/crazy paving followed by peri-lobular fibrosis combined with vascular thickening and bronchiectasis in a bilateral predominantly peripheral distribution with lower lobe predilection. On the other hand, lobar pneumonia and lung cavitation as well as diffuse, unilateral, or unifocal distributions were 
Table 4 Distribution of Lesions in COVID-19 pneumonia patients

\begin{tabular}{ll}
\hline Category and subcategory & No. of Patients (\%) \\
\hline Involved lungs and lobes & $588(68.2 \%)$ \\
Both lungs & $622(72.2 \%)$ \\
Right lung & $603(70 \%)$ \\
Left lung & $532(61.7 \%)$ \\
Right upper lobe & $504(58.5 \%)$ \\
Middle lobe & $609(70.6 \%)$ \\
Right lower lobe & $528(61.3 \%)$ \\
Left upper lobe & $489(56.7 \%)$ \\
Lingula & $578(67.1 \%)$ \\
Left lower lobe & \\
Lesion distribution & $626(72.6 \%)$ \\
Peripheral & $222(25.8 \%)$ \\
Peripheral and central & $558(64.7 \%)$ \\
Patchy & $16(1.9 \%)$ \\
Diffuse &
\end{tabular}

uncommon with the middle lobe and lingula being the least affected.

Similar to Carotti et al. [7], the most common associated imaging finding in COVID-19 was minimal lower lobar posterior pleural thickening.
In accordance with Salehi et al. and Kim et al. [14, 26], pleural effusion, pericardial effusion, and mediastinal lymphadenopathy were infrequently described associated findings.

It is worth noting that pleural and/or pericardial effusion were particularly reported in cases with advanced lung affection in the form of extensive multi-lobar consolidation and/or crazy paving. This agrees with Ojha et al.'s [6] declaration that pleural and pericardial effusion occur in advanced cases.

More than two-thirds of the mediastinal lymph nodes reported in our study were calcified, likely being a sequel of healed previous granulomatous infection. This may be attributed to the fact that tuberculosis is endemic in Egypt.

In few of our cases, a lung cyst was recognized among the other CT findings. A limited number of articles mentioned the presence of cyst(s) in cases of COVID-19; however, the etiology and relation to COVID-19 pneumonia are unclear [19, 22].

On searching the literature, none of the previous studies described thymic abnormalities in association with COVID-19. Nevertheless, thymic hyperplasia was identified in a considerable number of our cases with statistically significant young age and female predilection; most of them had no lung abnormalities on CT. The thymus is a lymphoid organ that plays a cardinal role in development of the immune system during childhood. It

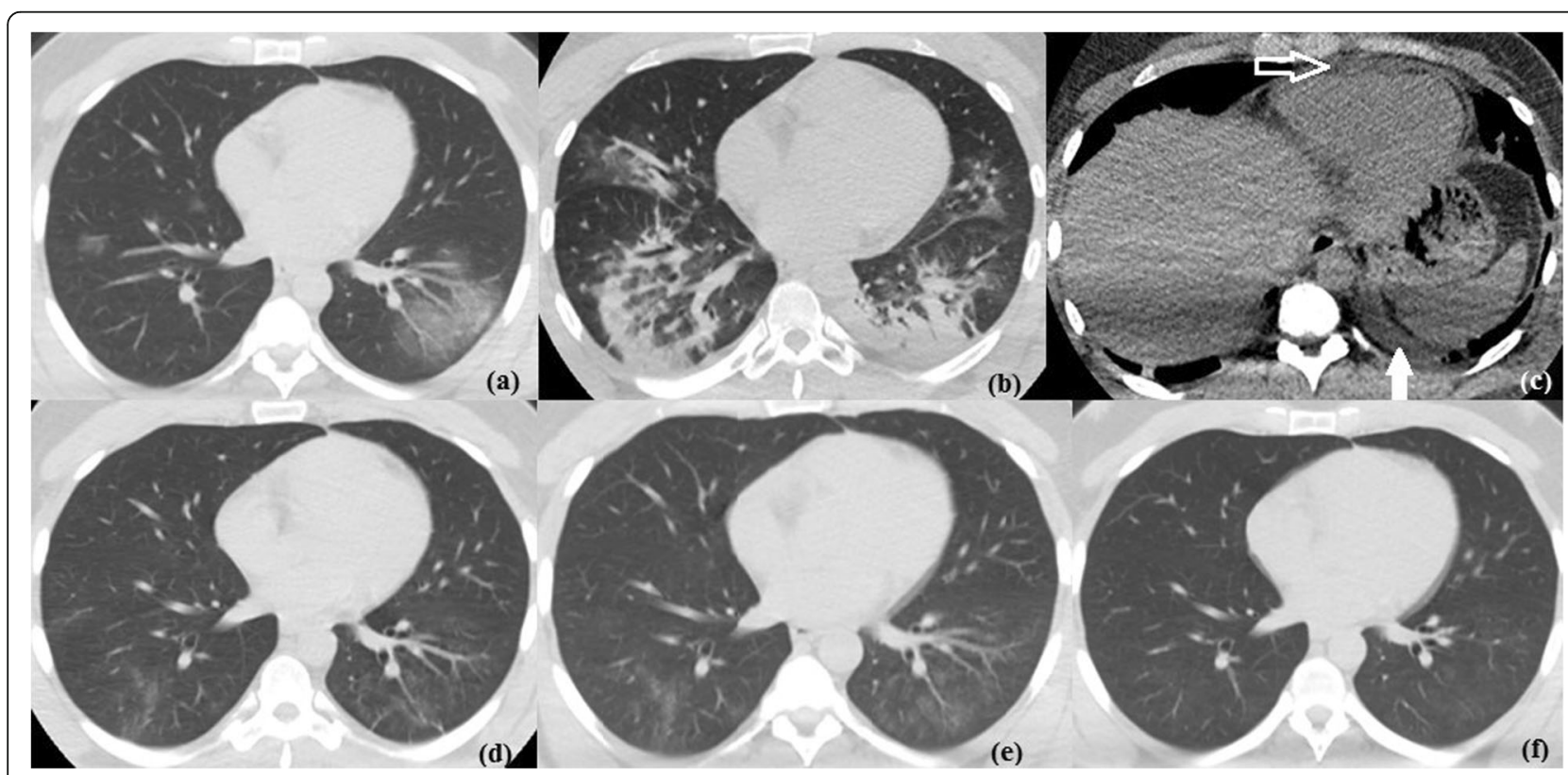

Fig. 5 Temporal CT changes in a 35-year-old male with COVID-19. a Initial non-contrast CT chest axial lung window showing multifocal bilateral predominantly peripheral patchy ground glass opacities with vascular thickening. b, c Follow up CT study after 7 days in axial lung and axial noncontrast mediastinal windows showing progressive course regarding the extent of lung involvement with bilateral predominantly peripheral patchy consolidation and air bronchogram associated with newly developed mild pericardial effusion (hollow arrow) and mild left pleural effusion (solid arrow). d-f Follow up images 16, 22, and 28 days respectively after initial CT showing gradual regressive course with only ill-defined faint GGO seen 
gradually involutes throughout maturation yet maintains the ability to re-grow (9). Hence, we suggest that thymic hyperplasia is an immune response to the viral infection; yet, further studies are warranted to validate this hypothesis.

Employing the previously illustrated RSNA Expert Consensus Statement, typical category was the most frequently encountered, while indeterminate and atypical categories were unusual. Correspondingly, CT can confidently diagnose COVID-19 in about $69 \%$ of cases. Taking into consideration the current pandemic and resources constraints (e.g., RT-PCR availability), the implementation of CT as a screening tool can be disputed.

Regarding follow up, progression was defined as an increase in the number, size, extent, or density of previously noted lesion(s) and/or development of new lesions, while regression would represent decrease in those finding(s). In this study, nearly half of the evaluated studies showed regression, one-third remained unchanged, and few cases showed progression.

On reviewing previously published studies, the percentage of cases with progressive CT findings ranged from 32 to $94.75 \%$. This wide range may be explained by the variability in study duration, number of cases, and time interval between initial and follow up CT studies $[13,14,16,17,19,20,27]$.

Twenty of our cases had persistent normal CT on follow up. In Xu et al.'s study [12], 75\% of cases had normal initial and follow up scans. Also, 13\% of patients evaluated by Zhang et al. [13] and $1.8 \%$ of those assessed by Guan et al. [16] remained negative on follow up. Consequently, we suggest that patients may not develop CT manifestations of pneumonia along the course of COVID-19 infection.

One of the major strengths of this study is its timing; as it was conducted early along the course of this not yet fully understood pandemic. Thereby, the whole radiological spectrum of COVID-19 was captured making this one of the most sensitive radiological studies of COVID19 cases in Egypt.

However, our study had some limitations including the unavailability of laboratory data and limited number of follow up studies. Furthermore, this is a hospital not a population-based study; therefore, sensitivity and specificity could not be calculated; yet, we could deduct the percentage of different $\mathrm{CT}$ imaging categories. It is thus recommended to perform future studies to confirm the generalizability of this study and similar ones.

\section{Conclusion}

In conclusion, although COVID-19 cannot be entirely excluded by chest $\mathrm{CT}$, it can be efficiently distinguished in more than two-thirds of cases; making CT a universally available, non-invasive, and rapid diagnostic tool for COVID-19.

\section{Abbreviations}

COVID-19: Coronavirus Disease 2019; GGO: Ground glass opacities; IRB: Institutional review board; NAT: Nucleic acid testing; RSNA: Radiological Society of North America; RT-PCR: Reverse-transcriptase polymerase-chainreaction; SARS-CoV-2: Severe acute respiratory syndrome coronavirus 2; SPSS: Statistical Product and Service Solutions; WHO: World Health Organization

\section{Acknowledgements \\ Not applicable.}

\section{Authors' contributions}

YYS, MMTF, EZN, SME, and SFT reviewed the CT images. SFT analyzed and interpreted the patient data. AAH did the statistical analysis. SFT and EZN wrote the manuscript and YYS reviewed it. All authors have read and approved the manuscript.

\section{Funding}

Not applicable (no funding was provided).

\section{Availability of data and materials}

The datasets used and/or analyzed during the current study are available from the corresponding author on reasonable request.

\section{Ethics approval and consent to participate}

Approval of the ethical committee of the 'Radiology department, Faculty of Medicine, Cairo University' was granted before conducting this prospective study; March 2020 - Reference number: not applicable. Written informed consent was obtained from all study participants or their authorized representatives.

\section{Consent for publication}

All patients included in this research gave written informed consent to publish the data contained within this study. If the patient was less than 16 years old, deceased, or unconscious when consent for publication was requested, written informed consent for the publication of this data was given by their parent or legal guardian.

\section{Competing interests}

The authors declare that they have no competing interests.

\section{Author details}

${ }^{1}$ Kasr Al-Ainy Faculty of Medicine, Cairo University, Al-Manial, Cairo 11559, Egypt. ${ }^{2}$ National Hepatology \& Tropical Medicine Research Institute (NHTMRI), 10 (A) Kasr El-Aini St, Cairo 11796, Egypt.

Received: 11 September 2020 Accepted: 4 November 2020 Published online: 27 November 2020

\section{References}

1. Li X, Zeng W, Li X, Chen H, Shi L, Li X, Xiang H, Cao Y, Chen H, Liu C, Wang $\mathrm{J}$ (2020) CT imaging changes of corona virus disease 2019 (COVID-19): a multi-center study in Southwest China. J Transl Med 18:1-8

2. Simpson S, Kay FU, Abbara S, Bhalla S, Chung JH, Chung M, Henry TS, Kanne JP, Kligerman S, Ko JP, Litt H (2020). Radiological Society of North America Expert Consensus Statement on Reporting Chest CT Findings Related to COVID-19. Endorsed by the Society of Thoracic Radiology, the American College of Radiology, and RSNA. Radiology: Cardiothoracic Imaging, 2(2), e200152.

3. Situation Report - 145. World Health Organization. https://www.who.int/ emergencies/diseases/novel-coronavirus-2019/situation-reports. Published June 13, 2020

4. Zu ZY, Jiang MD, Xu PP, Chen W, Ni QQ, Lu GM, Zhang LJ (2020) Coronavirus disease 2019 (COVID-19): a perspective from China. Radiology 296(2):E15-E25

5. Ye Z, Zhang Y, Wang Y, Huang Z, Song B (2020) Chest CT manifestations of new coronavirus disease 2019 (COVID-19): a pictorial review. European radiology 30(8):4381-4389

6. Ojha V, Mani A, Pandey NN, Sharma S, Kumar S (2020) CT in coronavirus disease 2019 (COVID-19): a systematic review of chest CT findings in 4410 adult patients. European Radiology 30(5):1-10 
7. Carotti M, Salaffi F, Sarzi-Puttini P, Agostini A, Borgheresi A, Minorati D, Galli M, Marotto D, Giovagnoni A (2020) Chest CT features of coronavirus disease 2019 (COVID-19) pneumonia: key points for radiologists. La Radiologia Medica 125:636-646

8. EMRO, 2020. http://www.emro.who.int/media/news/who-delegationconcludes-covid-19-technical-mission-to-egypt.html. Last access on July 2nd, 2020.

9. Nasseri F, Eftekhari F (2010) Clinical and radiologic review of the normal and abnormal thymus: pearls and pitfalls. Radiographics 30(2):413-428

10. Yang W, Yan F (2020) Patients with RT-PCR-confirmed COVID-19 and normal chest CT. Radiology 295(2):E3-E3

11. Asefi H, Safaie A (2020) The role of chest CT scan in diagnosis of COVID-19. Advanced Journal of Emergency Medicine 4(2s):e64-e64

12. Xu C, Xu Q, Du C, Li J, Hu Z, Yi Y (2020) Radiological follow-up of twelve COVID-19 patients with initially normal chest $C T$. Quantitative Imaging in Medicine and Surgery 10(5):1153

13. Zhang L, Kong X, Li X, Zhu J, Liu S, Li W, Xu C, Du H, Jing H, Xu J, Shi T (2020) CT imaging features of 34 patients infected with COVID-19. Clinical Imaging 68:226-231

14. Salehi S, Abedi A, Balakrishnan S, Gholamrezanezhad A (2020) Coronavirus disease 2019 (COVID-19): a systematic review of imaging findings in 919 patients. AJR Am J Roentgenol 215(1):87-93

15. Caruso D, Zerunian M, Polici M, Pucciarelli F, Polidori T, Rucci C, Guido G, Bracci B, de Dominicis C, Laghi A (2020) Chest CT features of COVID-19 in Rome, Italy. Radiology 296(2):e79-e85

16. Guan CS, Lv ZB, Yan S, Du YN, Chen H, Wei LG, Xie RM, Chen BD (2020) Imaging features of coronavirus disease 2019 (COVID-19): evaluation on thin-section CT. Academic radiology 27(5):609-613

17. Wang K, Kang S, Tian R, Zhang X, Wang Y (2020) Imaging manifestations and diagnostic value of chest CT of coronavirus disease 2019 (COVID-19) in the Xiaogan area. Clinical radiology 75(5):341-347

18. Güneyli S, Atçeken Z, Doğan H, Altınmakas E, Atasoy KÇ (2020) Radiological approach to COVID-19 pneumonia with an emphasis on chest CT. Diagn Interv Radiol 26(4):323-332

19. Shi H, Han X, Jiang N, Cao Y, Alwalid O, Gu J, Fan Y, Zheng C (2020) Radiological findings from 81 patients with COVID-19 pneumonia in Wuhan, China: a descriptive study. Lancet Infect Dis 20(4):425-434

20. Zhao W, Zhong Z, Xie X, Yu Q, Liu J (2020) Relation between chest CT findings and clinical conditions of coronavirus disease (COVID-19) pneumonia: a multicenter study. AJR Am J Roentgenol 214(5):1072-1077

21. Li Y, Xia L (2020) Coronavirus disease 2019 (COVID-19): role of chest CT in diagnosis and management. AJR Am J Roentgenol 214(6):1280-1286

22. Cheng Z, Lu Y, Cao Q, Qin L, Pan Z, Yan F, Yang W (2020) Clinical features and chest CT manifestations of coronavirus disease 2019 (COVID-19) in a singlecenter study in Shanghai, China. AJR Am J Roentgenol 215(1):121-126

23. Shang Y, Xu C, Jiang F, Huang R, Li Y, Zhou Y, Xu F, Dai H (2020) Clinical characteristics and changes of chest $C T$ features in 307 patients with common COVID-19 pneumonia infected SARS-CoV-2: A multicenter study in Jiangsu, China. Int J Infect Dis 96:157-162

24. Zhou S, Wang Y, Zhu T, Xia L (2020) CT features of coronavirus disease 2019 (COVID-19) pneumonia in 62 patients in Wuhan, China. AJR Am J Roentgenol 214(6):1287-1294

25. Wan S, Li M, Ye Z, Yang C, Cai Q, Duan S, Song B (2020) CT manifestations and clinical characteristics of 1115 patients with coronavirus disease 2019 (COVID19): A systematic review and meta-analysis. Acad Radiol 27(7):910-927

26. Kim H, Hong H, Yoon SH (2020) Diagnostic performance of CT and reverse transcriptase-polymerase chain reaction for coronavirus disease 2019: a meta-analysis. Radiology 296(3):e145-e155

27. Kuang Y, He S, Lin S, Zhu R, Zhou R, Wang J, Ying S, Li R, Lin H, Zhang Z, Pang P (2020). Clinical characteristics and CT manifestations of 143 hospitalized patients with 2019 novel coronavirus disease (COVID-19) outside Wuhan: a multi-center study in Taizhou City, Zhejiang, China. Zhejiang, China (3/14/2020).

\section{Publisher's Note}

Springer Nature remains neutral with regard to jurisdictional claims in published maps and institutional affiliations.

\section{Submit your manuscript to a SpringerOpen ${ }^{\circ}$ journal and benefit from:}

- Convenient online submission

- Rigorous peer review

- Open access: articles freely available online

- High visibility within the field

- Retaining the copyright to your article

Submit your next manuscript at $\boldsymbol{\nabla}$ springeropen.com 